\title{
How Authoritarian Leadership and Renqing Orientation Improve Tacit Knowledge Sharing
}

\author{
Zhen-Jiao Chen \\ Beijing Institute of Technology, Beijing, China \\ sharon2009@bit.edu.cn \\ Lin Jia \\ Beijing Institute of Technology, Beijing, China \\ Jialin87@bit.edu.cn
}

\begin{abstract}
Following an indigenous approach and fairness theory, we develop a theoretical model to investigate when and how authoritarian leadership will improve tacit knowledge sharing $(K S)$. Drawing on survey data from 309 Chinese employees, we examine whether authoritarian leadership will affect procedural fairness (PF) perception and interactional fairness (IF) perception, which in turn, will affect tacit KS; and examine whether leader renqing orientation (LRO) will moderate these mediation processes. Our data demonstrate that authoritarian leadership is negatively related to IF perception, which is positively related to tacit KS. Moreover, authoritarian leadership significantly increases tacit KS intention through PF perception only when LRO is high. Authoritarian leadership significantly decreases tacit KS intention through PF perception and IF perception only when LRO is low. Theoretical and practical implications are discussed.
\end{abstract}

\section{Introduction}

Economic globalization aggravates competition among enterprises worldwide, and knowledge sharing (KS) is a crucial strategic requirement for enterprises to compete successfully [13]. Knowledge shared within enterprises is commonly divided into tacit and explicit forms. Explicit knowledge can be codified into written documents and easily shared as independent entities [22]. Tacit knowledge is not codified and is only stored in individual people's brains [22]. Tacit KS is related to complex processes, such as role modeling and observation, in direct interpersonal contact [13]. Tacit $\mathrm{KS}$ intention and explicit KS intention are believed to be influenced by different factors [13]. The sharing of tacit knowledge is considered to be more difficult (because it is not codified) and more costly than explicit knowledge. Nevertheless, tacit knowledge is

\author{
Meng-Meng Song \\ Beijing Institute of Technology, Beijing, China \\ songmengmeng@163.com \\ Zhao-Hua Wang \\ Beijing Institute of Technology, Beijing, China \\ wangzhaohua@bit.edu.cn
}

considered to be more crucial to enterprise competitive advantage [13]. Thus, this study focuses on tacit KS intention.

$\mathrm{KS}$ is widely regarded as a social exchange between employees and enterprises [32]. Leaders who represent the enterprises are the social exchange partners of employees, and are important to employee KS [16]. However, most current KS studies adopt the etic lens (i.e., understanding a culture from the outside and using more cultural neutral or "objective" constructs) to examine the effect of western leadership on KS [16], only a few studies adopt the emic lens (i.e. understanding a culture from the inside) to investigate how Chinese indigenous leadership affects $\mathrm{KS}$ in the east. Understanding Chinese indigenous leadership is meaningful for both global scholars and practitioners given the globalization of business strategies. In the global economy, Chinese huge potential market has attracted substantial western enterprise to invest in China. Localization (e.g., employing Chinese managers and employees) is a sure avenue for these western enterprises to maximize profits [26]. Authoritarian leadership is an effective indigenous leadership style for Chinese employees because it fits into traditional values [8]. Although Authoritarian leaders, as important exchange partners of Chinese employees, have received minimal attention in KS studies, but will affect tacit KS among employees.

Fairness perception is decisive in maintaining a social exchange relationship [11]. Fairness is considered the fair input of direct supervisors to the social exchange relationship with their subordinates [11] Employees use fairness information to infer the degree of trust that they should place in their leaders (exchange partners) and to decide whether to engage in social exchange relationships with their leaders [11]. Tacit $\mathrm{KS}$ is about providing knowledge to organizations while expecting reciprocity [32]. The 
perceived fairness of the management practices of leaders will definitely encourage employees tacit KS. Despite the importance of fairness, the extant $\mathrm{KS}$ literature has paid little attention on its effects [28]. This gap has elicited our first question: Does authoritarian leadership affect employee fairness perception of management practices, which in turn, influence employee tacit KS intention in the Chinese context?

Furthermore, it is well-known that guanxi (the traditional Chinese concept of personal relationships) culture is deeply rooted in China and has a great impact on the behaviors of Chinese employees [17]. Previous scholars [15] have suggested that many IT-based KM initiatives have failed in China because IT cannot function as a substitute for the incentive effects of human relationships and social connectedness on KS. Therefore, exploring socio-cultural factors related to human relationships and social connectedness is important in Chinese KM research [15, 23].

Thus, we propose LRO, as a form of social exchange norm, should regulate the exchange relationship between authoritarian leadership and employee tacit KS. It is well-known that "In-group" collectivism and guanxi culture are deeply rooted in China. Face and renqing represent two facets of guanxi [17]. Face refers to maintaining a positive public image. LRO refers to the tendency of a leader to care for subordinates emotional responses and to follow a form of social exchange norm in exchanging with "in-group" subordinates [17]. Several scholars have adopted the emic perspective to examine how $\mathrm{KS}$ is affected by face, including face giving, face gaining, and face maintenance $[15,23,30]$, but have ignored the effect of renqing, which is notably an important exchange rule in Chinese society. Thus, our second question explores whether the renqing orientation of leaders moderates the effect of authoritarian leadership on employee tacit $\mathrm{KS}$ intention through fairness perception.

To address the aforementioned questions, we adopt the tenets of fairness theory $[11,19]$ to conceptualize the interweaving of authoritarian leadership (exchange partner), LRO (exchange norm), fairness perception (psychological process of exchange), and employee tacit KS intention (reciprocal intention). Fairness theory explains how fairness perceptions are formed and what outcomes will be affected by various fairness dimensions $[11,19]$. On the one hand, fairness theory identifies a set of procedural rules of management and various interpersonal treatments as predictors of procedural fairness and interactional fairness in leader decision-making processes. On the other hand, this theory claims that different fairness dimensions are beneficial to various outcomes, such as organizational citizenship behavior (OCB) and KS [11]. OCB is an extra-role employee behavior $[16,25]$.
According to fairness theory, if the management process is deemed as a fair process of decisionmaking or fair interpersonal treatment, subordinates will trust the exchange relationship with their leaders and reciprocate such fair treatment by engaging in discretionary behaviors, such as OCB and tacit KS [7, 11]. Our study proposes that authoritarian leadership, with rigorous control, dominance and highperformance orientation, is negatively related to $\mathrm{PF}$ perception and IF perception, which in turn, are positively related to employee tacit KS intention. Moreover, LRO is expected to moderate the aforementioned mediating processes. Authoritarian leadership is expected to increase tacit KS intention through PF perception only when LRO is high. Authoritarian leadership is expected to decrease tacit KS intention through PF perception and IF perception when LRO is low. We argued that only employees who completely obey and make contribution to high performance (e.g., tacit KS) are deemed as possible "in-group" members by authoritarian leaders. LRO refers to the tendency of authoritarian leaders to care for subordinate emotional responses and to allocate better resources to reciprocate "in-group" subordinates who have made contribution to group performance (e.g., tacit KS). This practice leads to employee PF perception and IF perception, and further improves tacit $\mathrm{KS}$ intention.

The reminder of this paper is organized as follows. Section 2 provides a comprehensive literature review and the theoretical background. Section 3 presents the research model and hypotheses. Section 4 describes the research method, and Section 5 reports the data analysis and its findings. Section 6 discusses the theoretical and practical implications.

\section{Literature review}

\subsection{Fairness theory}

Fairness theory is derived from organization behavior field and is adopted to explain social exchange relationship in both the workplace and various information system (IS) settings [for a review, see 7, 11]. Fairness theory divides organizational fairness into three dimension: distributive fairness (DF), PF and IF [11]. We focus on PF and IF, given that recent meta-analyses show that these two dimensions are more directly related to OCB [6, 29]. $\mathrm{KS}$ can be regarded as a form of OCB [25]. Employee PF perception refers to the perceived fairness of resources allocation and decision-making procedures of leaders. Employee IF perception refers 
to the perceived fairness of the interpersonal treatment of their leaders in workplaces [11, 19].

Fairness theory indicates that PF perception is increased by fair decision procedures characterized by transparency, voice, neutrality, bias suppression, consistent standard, and ethnicity [19]. Meanwhile, IF perception is developed by the fair treatment of leaders that is characterized by respect, propriety, and truthfulness [11, 12]. Furthermore, fairness theory claims that fairness perception causes employees to redefine leader-member relationships as a trustful social exchange, thereby encouraging reciprocal behaviors (e.g., OCB) [11, 19]. Subsequent empirical studies on fairness have confirmed fairness theory. PF perception is found to be significantly increased by various fair procedural characteristics, such as transparency, voice, and consistency [for a review, see 7]. IF perception is found to be significantly increased by the fair treatment of leaders, such as two-way communication, respect, organizational support, and leader-member exchange [12]. PF and IF are found to increase in-role performance and ex-role OCB in the workplace, as well as user trust, user satisfaction and continuous use intention in various IS settings [7].

However, the aforementioned fairness research mainly identified western socio-cultural factors from western theories as preditors of fairness, and focused minimal attention on the effect of indigenous factors in the eastern context $[15,23]$. Furthermore, although KS is regarded as a typical outcome of the social exchange between employers and employees, only a few studies have examined fairness-KS intention relationship [28]. Our study fills in these research gaps. We adopt fairness theory to explore whether Chinese authoritarian leadership affects the PF perception and IF perception of employees, which in turn, influence their tacit KS intention. Moreover, we identify LRO, an indigenous social exchange rule in China, as a moderator that regulates the indirect effects of authoritarian leadership on tacit KS intention through PF and IF.

\subsection{Authoritarian leadership}

Authoritarian leadership refers to a leader having absolute control over his/her subordinates and requiring unquestionable obedience [8]. Authoritarian leadership originates from the Chinese patriarchal tradition and the Confucian ethics of hierarchical order. It is characterized by domination, belittling the abilities of subordinates, building a lofty image for the leader and instructing subordinates to achieve high group performance. The subordinates will be socialized to follow a hierarchical order [8, 29]. Authoritarian leadership is one of the three dimensions of paternalistic leadership (PL). The other two dimensions, i.e., benevolent leadership and moral leadership, are welldocumented to improve work outcomes, whereas prior studies show that authoritarian leadership is a two edged sword; that is, it has both positive and negative effects on work outcomes (e.g., OCB, performance) $[4,5,8,29]$. Thus, we focus on authoritarian leadership to interpret the "black box" of its inconsistent effects.

Research on authoritarian leadership has flourished recently with the development of Chinese economy [4]. To interpret the inconsistent authoritarianism-outcome relationships, one line of research adopts the "mediation approach" to demonstrate that the authoritarianism-outcome relationships are mediated by mediators, such as trust, organization esteem, fear and resentment, and leadermember exchange [4, 8, 29, 31]. Another line of research adopts "moderation approach" to show that relationships are regulated by moderators, such as benevolent leadership, information sharing, subordinate's dependence, and authority orientation [8, 10, 31]. Although leader-member interaction can be elaborated from the perspective of social exchange, the aforementioned research did not identify the psychological mechanisms involved in exchange as mediators in authoritarianism-outcome relationships. Therefore, we propose two such mediators: PF perception and IF perception. Moreover, we also identify a moderator, namely, renqing orientation (Chinese social exchange rule), to regulate the mediation processes.

\subsection{Renqing orientation}

The principle of renqing stems from the Confucian ethic of "relationalism" and the Chinese guanxi (i.e., face and favor) culture. Relationalism refers to a rule of favoring intimates with whom individuals have good relationships and will exchange favors. Face and favor theory posits that renqing has two meanings [17]. First, it refers to following an exchange norm to provide intimates with resources as gifts to maintain social exchange relationships [17]. Second, renqing refers to the emotional response triggered by a situation, such as happiness, anger, hate, love and desire [17]. A person who practices renqing should understand and sympathize with the emotional responses of others to different situations, or even cater to their desires and avoid whatever they resent [17]. Thus, the present study defines the renqing orientation of leaders as their tendency to allocate more resources to intimate subordinates with whom they have a social exchange relationship and their tendency to care for their subordinate emotional responses. 
The popularity of Chinese LRO is reflected by the prevalence of the "guanxi practices" of Chinese enterprises. For example, it is common for many Chinese leaders to give more rewards and promotion opportunities to their intimate subordinates or make decision of resource allocation based on both contribution and guanxi [6].

Despite renqing is possibly to encouraging social exchange and reciprocity, prior literature denotes its double nature $[6,15,23]$. Repeated behavior becomes normative. Renqing orientation is popular among Chinese leaders; hence, guanxi-based resources exchange is regarded as fair by some employees in China; however, other employees prefer a contributionbased resources allocation system and deem renqing as unfair [6]. In line with these arguments, the guanxi network is found to increase performance and KS [23], whereas group guanxi is found to decrease PF [6]. Guanxi include face and renqing [17]; however, the KS literature has mainly focused on face and gave minimal attention to renqing $[15,23,26]$. Thus, our study examines the moderating effect of LRO on tacit KS intention.

\section{Theoretical model and hypotheses}

Fairness perception is supposed to mediate the relationship between authoritarian leadership and tacit $\mathrm{KS}$ intention in this study. Fairness theory tenets provide a compelling explanation. As noted in the literature review, fairness theory identifies various procedural rules (e.g., voice, transparency, neutrality) and interpersonal treatment as predictors of PF perception and IF perception, respectively [11, 12, 19]. This theory also explains how PF perception and IF perception improve work outcomes by fostering trust and commitment [11].

Authoritarian leaders strictly control resourceallocation decision processes by withholding detailed information regarding criteria and procedures, and refusing to engage in communication. They simply inform their subordinates regarding their decisions and require complete obedience from them [8, 29]. Such behavior decreases the neutrality and transparency of a decision process as well as discourages employee voice $[13,19,31]$. A decision process without neutrality, transparency, and voice will cause employees to perceive procedural unfairness [19]. A vast amount of empirical evidence shows that authoritarian leadership is negatively related to voice and transparency [3, 31], which are positively related to PF perception $[7,11,19]$.

Furthermore, perceived procedural unfairness increases the sense of uncertainty of employee, because unfair decision procedures are unpredictable and outside of the control of employees [11]. The sense of uncertainty reduces the commitment of employees to exchange with their leaders, thus, their intention to reciprocate by sharing tacit KS is decreased [20]. This argument is consistent with the prior empirical finding that PF perception is positively related to organizational commitment, which in turn, is positively related to tacit $\mathrm{KS}[20,25]$. Hence, we propose the following hypothesis (see Figure 1):

Hypothesis 1a: PF perception mediates the negative relationship between authoritarian leadership and tacit $K S$ intention.

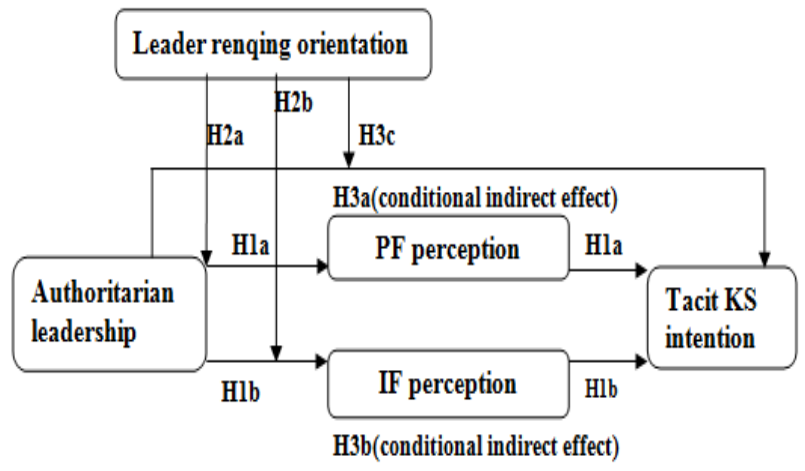

Figure 1. Research model

Authoritarian leaders demonstrate strict control and dominance, which are interpreted as regarding their subordinates as incompetent [31].To build a lofty image and emphasize absolute authority, authoritarian leaders are less likely to value and respect the contributions of their subordinates [4, 31]. Fairness theory states that the low-quality treatment of a leader decreases employee perception of IF in the workplace $[11,12]$. In line with this argument, prior studies found that authoritarian leadership significantly reduced employee self-esteem and IF perception [4, 29].

Interpersonal unfairness in leader-member interaction frequently causes employees to feel anger and anxiety [29]. These negative emotions reduce the trust of employees on their exchange partners (the leaders), thereby discouraging tacit KS intention. Zhang and colleague [31] found that authoritarian leadership is negatively related to leader-member exchange. Wu et al. [29] found that IF perception was positively related to trust and OCB. KS is a form of OCB. We propose the following hypothesis based on the preceding argument:

Hypothesis 1b: IF perception mediates the negative relationship between authoritarian leadership and tacit KS intention.

We propose that the LRO can moderate the negative effects of authoritarian leadership on the PF 
perception of subordinates. Authoritarian leaders are achievement-oriented $[4,8]$. They requires their group to achieve the best performance in an organization by imposing strict control, setting rules, determining job responsibilities, issuing punishment and rewards [4, 8]. Hence, only subordinates who obey the rules and contribute to group performance (e.g., tacit KS) will become the "in-group" members of the leaders and will establish good relationships with them $[4,8]$.

First, an authoritarian leader with high renqing orientation is expected to follow a social exchange norm and grant better resources to "in-group" subordinates who have made valuable contributions to group performance, such as tacit KS. That is, an authoritarian leader with high renqing orientation is expected to use contribution-based resources allocation criteria conduct social exchange with his/her subordinates, which increases the transparency and neutrality of his/her resource-allocation decision processes [11, 12, 19]. Second, an authoritarian leader with high renqing orientation is expected to express more emotional concern to subordinates who share tacit knowledge. The leaders will understand the emotional response of these subordinates, or even cater to their desires [17]. Hence, the leaders is more likely to listen and respect the ideas of these subordinates, who make good contribute to group performance, thereby improving the opportunity of subordinates to have their voice heard during decision processes. In summary, an authoritarian leader with high renqing orientation will use contribution-based resources allocation criteria and express more emotional concern (e.g., listen and respect) to reciprocate these "in-group" subordinates who share tacit knowledge to their groups. This situation increases the voice, neutrality, and transparency in the resourcesallocation decision processes, and thereby improving subordinate perception of PF.

By contrast, an authoritarian leader with low renqing orientation will not follow the renqing rule of reciprocating subordinates who made contributions with more resources or emotional concern. Instead, their contributions are sometimes devalued in favor of building a lofty image for the leader. In the resourcesallocation decision process, the unilateral actions of the leader are characterized by strict control and dominance, thereby making the process subjective, non-transparent, and without neutral criteria and voice. This situation decreases subordinate PF perception.

Hypothesis 2a: LRO moderates the relationship between authoritarian leadership and PF perception, such that the relationship is positive when LRO is high; and the relationship is negative when LRO is low.
The dominance and strict control of an authoritarian leader are often interpreted by subordinates as belittling and disrespectful, which may trigger anxiety and anger [29, 31]. When LRO is high, the leaders will implement measures to avoid stimulating negative emotions among his/her subordinates [17]. Employees may interpret control as a mentoring attempt or an achievement-oriented approach, and thus, may react positively [4]. That is, the high renqing orientation of a leader is expected to counteract the negative effect of his/her authoritarianism on subordinate IF perception. By contrast, authoritarian leaders with low renqing orientation will not understand or take actions to avoid invoking negative emotions among his/her subordinates. Their authoritarianism should be regarded as poor interpersonal treatment that decreases subordinate IF perception.

Prior research has denoted that simultaneously utilizing fear and favor improve work attitudes [8]. Zhou [33] also argued that paternalistic control, i.e., authoritarian control and benevolence, may increase group creativity in China. Chan et al. [4] found that benevolent leadership moderated the negative effect of authoritarian leadership on employee self-esteem and OCB. Hence, we hypothesize:

Hypothesis 2b: LRO moderates the relationship between authoritarian leadership and IF perception, such that the relationship is nonsignificant when LRO is high; but the relationship is negative when LRO is low.

Furthermore, we propose that authoritarian leadership is negatively related to PF perception and IF perception, which in turn, decreases tacit $\mathrm{KS}$ intention when leaders have low renqing orientation. By contrast, if leaders have high renqing orientation, then a performance-oriented authoritarian leadership may be positively associated with PF perception, which in turn may uphold tacit KS. We also contend that LRO may buffer the negative effects of authoritarian leadership on the tacit KS intention of subordinates because it makes such leadership less threatening to the psychological experience of subordinates in their social exchange with their leaders (in our case, PF perception and IF perception). In summary, we believe that the mediation of PF perception and IF perception on the relationship between authoritarian leadership and tacit KS intention may vary according to whether LRO is high or low. We also believe that LRO may impact the relationship of authoritarian leadership with tacit KS intention relationship in the same way it influences 
the relationship of authoritarian leadership with PF perception. Therefore, we hypothesize the following:

Hypothesis 3a: Authoritarian leadership increases the tacit KS intention through PF perception when LRO is high. By contrast, it decreases tacit KS intention through PF perception when LRO is low.

Hypothesis 3b: Authoritarian leadership decreases tacit KS intention through IF perception only when LRO is low rather than when it is high.

Hypothesis 3c: LRO moderates the relationship between authoritarian leadership and tacit KS intention, such that the relationship is positive when LRO is high; but the relationship is negative when LRO is low.

\section{Methodology}

\subsection{Data collection procedure}

A simple random sampling procedure was performed to select two enterprises located in Beijing in mainland of China to conduct the survey. The first author and a research assistant briefly introduced two senior managers of the human resource departments of the two enterprises about the purposes and procedures of the study. Then, the two managers helped us identify participants who were voluntarily involved in the survey. Questionnaires were administered to 400 participants from the two companies. All participants received questionnaires and survey introduction cover letters prepared by the researchers. Among which, a total of 309 usable responses were gathered, which represented a response rate of 77 percent. Among 309 participants, 68.30 percent were male; 86.1 percent had college or above education; 80.6 percent were from 21 to 40 years old; 68.3 percent were junior staff and 31.7 percent were managers (see Table 1). Table 1 presents the demographic information of the respondents.

Table 1. Demographics summary

Demographics summary $(\mathrm{n}=309)$

\begin{tabular}{lll}
\hline & Number & Percentage \\
\hline Gender & & \\
Male & 211 & $68.30 \%$ \\
Female & 98 & $31.70 \%$ \\
Educational level & & \\
Secondary School & 43 & $13.90 \%$ \\
College & 63 & $20.40 \%$ \\
Bachelor's & 159 & $51.50 \%$ \\
Master's or above & 44 & $14.20 \%$
\end{tabular}

\begin{tabular}{lll}
$\underline{\text { Age }}$ & & \\
$\leq 20$ & 2 & $0.60 \%$ \\
$21-40$ & 249 & $80.60 \%$ \\
$41-60$ & 54 & $17.50 \%$ \\
$>60$ & 4 & $1.30 \%$ \\
Job level & & \\
Staff & 211 & $68.30 \%$ \\
Junior manager & 68 & $22.00 \%$ \\
Middle manager & 26 & $8.40 \%$ \\
Senior manager & 4 & $1.30 \%$ \\
Enterprise & & \\
Enterprise 1 & 170 & $55 \%$ \\
Enterprise 2 & 139 & $45 \%$ \\
\hline
\end{tabular}

\subsection{Measures}

The items for all studied variables were adopted from the English literature. The survey was executed in China, and thus, the questionnaire was translated into Chinese via back-translation. The measures for the studied variable included 19 questions. All the items (see the Appendix) were measured by a fivepoint Likert scale; ranging from $1=$ strongly agree to $5=$ strongly disagree.

Specifically, six items for the authoritarian leadership construct were adopted from Chou and colleague [10] research. Two items used to measure $\mathrm{PF}$ perception were from Masterson et al. [21] research. Four items were adopted from Bies and Moag [1] study to measure IF perception. LRO was measured by using an abbreviated two-item scale adapted from the renqing scale of Cheung et al. [9] study. Tacit KS intention was measured using three items adopted from Bock et al.'s [2] study. OCB was measured using an abbreviated two-item version from Masterson and colleagues' [21] research to reflect the employee discretionary behaviors.

\section{Data analysis and findings}

LISREL (version 8.70) [18] and SPSS (version 17.0) were used to conduct the data analysis.

\subsection{Measurement model}

The Cronbach's alpha of all studied variables ranges from 0.70 to 0.91 (see Table 2). We conducted factor analysis with principal components analysis and varimax rotation to test the convergent and discriminant validity of the measures. The results in Table 2 showed that all loadings were significant 
$(p<0.01)$, and the items within the same construct correlated highly amongst themselves $(>0.70)$. Moreover, the items loaded more highly on their intended constructs than on other constructs. The results indicated that the convergent and discriminant validity of the measures were satisfactory.

Table 2. Validity and reliability of all questions

\begin{tabular}{|c|c|c|c|c|c|c|}
\hline \multirow{2}{*}{$\begin{array}{l}\text { Ques- } \\
\text { tion }\end{array}$} & \multicolumn{6}{|c|}{ Factor } \\
\hline & 1 & 2 & 3 & 4 & 5 & 6 \\
\hline AL 1 & 0.66 & 0.19 & 0.08 & 0.02 & 0.01 & 0.03 \\
\hline AL 2 & 0.67 & -0.26 & -0.12 & -0.06 & 0.20 & 0.26 \\
\hline AL 3 & 0.81 & -0.19 & 0.00 & -0.08 & 0.04 & 0.16 \\
\hline AL 4 & 0.82 & -0.14 & -0.03 & 0.06 & 0.08 & 0.06 \\
\hline AL 5 & 0.83 & -0.17 & 0.02 & -0.02 & 0.01 & 0.07 \\
\hline AL 6 & 0.70 & 0.05 & 0.16 & 0.08 & -0.14 & 0.11 \\
\hline LRO1 & 0.13 & 0.01 & 0.09 & 0.01 & -0.09 & 0.89 \\
\hline LRO2 & 0.40 & -0.05 & -0.04 & -0.09 & -0.01 & 0.76 \\
\hline PF 1 & 0.01 & 0.25 & 0.17 & 0.08 & 0.88 & -0.02 \\
\hline PF 2 & 0.10 & 0.17 & 0.29 & 0.14 & 0.84 & -0.09 \\
\hline IF 1 & -0.07 & 0.84 & 0.20 & 0.11 & 0.15 & 0.03 \\
\hline IF 2 & -0.06 & 0.90 & 0.10 & 0.10 & 0.13 & -0.03 \\
\hline IF 3 & -0.10 & 0.89 & 0.11 & 0.14 & 0.14 & -0.02 \\
\hline IF 4 & -0.11 & 0.75 & 0.20 & 0.08 & 0.04 & -0.05 \\
\hline TKSI1 & 0.08 & 0.18 & 0.84 & 0.06 & 0.15 & 0.03 \\
\hline TKSI2 & 0.07 & 0.12 & 0.84 & 0.10 & 0.15 & 0.11 \\
\hline TKSI3 & -0.02 & 0.24 & 0.82 & 0.09 & 0.13 & -0.09 \\
\hline OCB1 & 0.01 & 0.15 & 0.10 & 0.94 & 0.09 & -0.05 \\
\hline OCB2 & 0.02 & 0.19 & 0.13 & 0.93 & 0.11 & -0.02 \\
\hline \multicolumn{7}{|c|}{ Eigenvalue } \\
\hline & 5.08 & 3.99 & 1.58 & 1.52 & 1.27 & 1.00 \\
\hline \multicolumn{7}{|c|}{ Variance explained (\%) } \\
\hline & 26.7 & 21.0 & 8.30 & 8.00 & 6.68 & 5.24 \\
\hline \multicolumn{7}{|c|}{ Cumulative variance (\%) } \\
\hline & 26.7 & 47.7 & 56.0 & 64.0 & 70.7 & 75.9 \\
\hline \multicolumn{7}{|c|}{ Cronbach's alpha } \\
\hline & 0.86 & 0.91 & 0.85 & 0.92 & 0.86 & 0.70 \\
\hline
\end{tabular}

Note. $\mathrm{AL}=$ authoritarian leadership, $\mathrm{TKSI}=$ tacit $\mathrm{KS}$ intention

\subsection{Common method bias}

We conducted the chi-square difference test by using LISREL 8.70 to compare the six-factor model with five alternative models that increase in complexity. If there is common method bias in our data, a simple model will fit the data as well as a more complex model [24]. However, the results in Table 3 showed that the fit of the six-factor model was significantly better than each of the five alternative models. The results minimized the possibility of common method bias [24].

Table 3. Measurement model comparison

\begin{tabular}{lcccccc}
\hline Model & df & $\chi 2$ & CFI & GFI & RMSEA & $\triangle \chi^{2}$ \\
\hline 6-factor & 137 & 258 & 0.97 & 0.92 & 0.05 & \\
5-factor & 142 & 347 & 0.96 & 0.89 & 0.07 & $89^{*}$ \\
4-factor & 146 & 1591 & 0.77 & 0.65 & 0.18 & $1332^{*}$ \\
3-factor & 149 & 2117 & 0.70 & 0.58 & 0.21 & $1859^{*}$ \\
2-factor & 151 & 2373 & 0.65 & 0.55 & 0.22 & $2115^{*}$ \\
1-factor & 152 & 2808 & 0.59 & 0.51 & 0.24 & $2550^{*}$
\end{tabular}

Note. CFI=comparative fit index, GFI $=$ Goodness of Fit Index, and RMSEA $=$ root mean square error of approximation.

\subsection{Hypotheses testing}

Age, job level, gender, education, enterprise and $\mathrm{OCB}$ were included as control variables, as previous research has identified them as predictors of KS intention [16]. OCB was divided into supervisordirected OCB and organization-directed OCB. This study focuses on the latter given that KS can be regarded as a social exchange between employees and enterprises [32] and organization-directed OCB is expected to be more directly related to KS.

We used Hayes' [14] bootstrapping approach (n boots $=1000 ; 95 \%$ Bias corrected confidence interval to test the mediation (H1a and H1b) (i.e., indirect effect) and conditional indirect effects (H3a and H3b). Bootstrapping was found to be the most powerful methods to detect mediation and conditional indirect effects [14] and it has been used by lots of studies on organizational behavior and IT-based social media [for a review, see 27]. A confidence interval must not contain a zero to assume a significant mediation or conditional indirect effects [14].

The bootstrapping analysis found that authoritarian leadership had no effect on PF perception ( $\beta=0.080$, ns.) $[95 \%$ Bias corrected confidence interval (BC 95\% CI); -0.057, 0.216], which in turn, significantly improved tacit KS intention $(\beta=0.233, \mathrm{p}<0.05)$ [BC 95\% CI; 0.155, 0.312]. Authoritarian leadership significantly decreased IF perception $(\beta=-0.177, \mathrm{p}<0.05)$ [BC $95 \% \mathrm{CI} ;-0.273,-0.081]$, which in turn, significantly improved tacit KS intention $(\beta=0.261, p<0.05)$ [BC $95 \%$ CI; 0.152, 0.370]. Thus, authoritarian leadership exerted a significant and indirect effect on tacit KS intention though IF perception $(\beta=-0.046, p<0.05)$ [(BC 95\% CI $) ;-0.098,-0.019]$ rather than $\mathrm{PF}$ perception $(\beta=0.019$, ns.) $[\mathrm{BC} 95 \% \mathrm{CI} ;-0.016$, $0.060]$. Hypothesis $1 \mathrm{~b}$ was supported, but Hypothesis 1a was not supported. 
Moderated multiple regression was used to test Hypotheses 2a, 2b and 3c. As predicted, LRO significantly moderated the effects of authoritarian leadership on PF perception $(\beta=0.25, p<0.01)$, IF perception $(\beta=0.15, \mathrm{p}<0.01)$, and tacit KS intention $(\beta$ $=0.18, \mathrm{p}<0.01)$. Thus, Hypotheses $2 \mathrm{a}, 2 \mathrm{~b}$ and $3 \mathrm{c}$ were supported.

Finally, the bootstrapping analysis (see Table 4) found that the indirect effect of authoritarian leadership on tacit KS intention through PF perception was significant and positive only when LRO was high $(+1$ SD) $(\beta=0.105 ; \mathrm{p}<0.05)$ [BC 95\% CI; 0.047, 0.190]. When LRO was low (-1 SD), the indirect effect of authoritarian leadership on tacit KS intention through PF perception was significant and negative $(\beta=-0.043$; $\mathrm{p}<0.05$.) [BC 95\% CI; -0.097, -0.008]. Hypothesis 3a was supported. As expected, the indirect effect of authoritarian leadership on tacit KS intention through IF perception was significant and negative only when LRO was low $(-1$ SD) $(\beta=-0.085, \mathrm{p}<0.05)$ [BC 95\% CI; $0.159,-0.036]$ rather than when it was high $(+1 \mathrm{SD})$ $(\beta=-0.009$, ns. $)[\mathrm{BC} 95 \% \mathrm{CI} ;-0.056,0.021]$. Hypothesis $3 \mathrm{~b}$ was also supported. In total, the conditional indirect effect model explains about $31 \%$ of the variance of employee tacit KS intention.

Table 4. Testing the conditional indirect effects

\begin{tabular}{lcccc}
\hline & \multicolumn{2}{c}{ LRO } & & \multicolumn{2}{c}{$\begin{array}{c}\text { Tacit KS } \\
\text { intention }\end{array}$} \\
\hline & (moderator) & \multicolumn{3}{c}{ BC 95\% CI } \\
\cline { 2 - 5 } & & \multicolumn{1}{c}{$\beta$} & Lower & Upper \\
\hline PF & $-1 S D$ & -0.043 & -0.097 & -0.008 \\
(mediator) & $+1 \mathrm{SD}$ & 0.105 & 0.047 & 0.190 \\
IF & $-1 \mathrm{SD}$ & -0.085 & -0.159 & -0.036 \\
(mediator) & $+1 \mathrm{SD}$ & -0.009 & -0.056 & 0.021 \\
\hline
\end{tabular}

\section{Discussion}

This study adapted fairness theory and indigenous research perspective to examine how two Chinese indigenous factors, i.e., authoritarian leadership and renqing orientation, affect employee tacit KS intention. Our findings show that the mediation effects of PF perception and IF perception on the relationship between authoritarian and tacit KS intention vary according to whether LRO is high or low. This study is pioneering with respect to combine western fairness theory with Chinese indigenous management factors to explain employees KS intention in the Chinese context. Inconsistent with $\mathrm{H} 1 \mathrm{a}$, results show authoritarian leadership has no significant relationship with PF perception. This finding is also in agreement with some prior studies which found authoritarian leadership has no significant relationship with compliance, performance and OCB $[8,29]$. The possible reason for the unexpected finding could be explained by the confirmation of $\mathrm{H} 2 \mathrm{a}$. The main effect of authoritarianism on PF perception is ambiguous because it varies across the level of LRO. Except for $\mathrm{H} 1 \mathrm{a}$, all the hypotheses are supported.

\subsection{Theoretical implications}

Our findings have at least three important theoretical implications. First, based on indigenous approach, we identify authoritarian leadership as an important predictor of tacit $\mathrm{KS}$ intention. The Chinese construct is rarely examined in a Western context and should be considered in future theorizing of knowledge management, as no theory can be universal if it ignores significant indigenous constructs of a large national group such as the Chinese [23]. Furthermore, the authoritarian leadership is similar to the autocratic leadership in the west [29]. Chinese indigenous constructs may be important in non-Chinese culture and contribute to universal management theories.

Second, we adopt fairness theory from organizational behavior field to develop a theory framework and confirm the rationale of fairness theory in the field of KM. Our study also contributes to general literature on KM. We identify the important role of fairness perception in $\mathrm{KS}$ and introduce a relatively novel antecedent to KM field. Consistent with fairness theory, our findings demonstrate that the authoritarian leadership affects the formation of fairness perception, which in turn, significantly affect outcomes, such as tacit KS intention. Our research pioneers this approach in Chinese context, and enlightens future researcher to explore other independent variables, which can improve the formation of fairness perception and further increases tacit $\mathrm{KS}$ intention.

Third, our research shows that LRO can regulate the effects of authoritarian leadership on tacit KS intention, which demonstrates the utility of the interactionist approach to KM research. Our study incorporates LRO as a novel moderator into the conceptual model of KM.

\subsection{Practical implications}

During the process of globalization, some people suggested abandoning the indigenous management styles because of their negative effects [4]. However, in today's increasingly integrated global economy, Chinese huge potential market has attracted substantial western enterprise. Localization (e.g., 
employing Chinese managers and employees) is a sure avenue for these western enterprises to succeed in China [26]. Hence, it is pivotal for global managers to understand the two sides of some Chinese management styles (e.g., authoritarianism and renqing rule) so that can guide them to improve desirable outcomes (e.g., tacit KS). Our study tends to enlighten global managers in these aspects.

Our results have three major practical implications. First, our study demonstrates that authoritarian leadership is a two-edged sword and can be effective in specific contexts. Our findings show that authoritarian leadership is positively associated with employee tacit $\mathrm{KS}$ intention through employee PF percpetion when LRO is high. This finding suggests that managers can adopt authroritarian leadership when they also have high renqing orientation to promote employee tacit KS intention. The "carrot -and- big stick" policy can stimulate the tacit KS intention of employees. However, we also suggest that to avoid decreasing employee tacit KS intention, managers should reduce their authoritarinism when they have low renqing orientation. Furthermore, such managers should explore contervailing management practices to ameliorate their controlling behavior and thus, avoid unfairness perceptions among their subordinate.

Second, our research suggests that when renqing orientation is combined with high-performance oriented authoritarianism, the interaction between these two variables can be benificial to improve employee fairness perception and tacit KS intention. Although some experts suggest that renqing orientation can lead to corruption and unfairness[6]. Our research implies that authoritarian leaders should cultivate renqing orientation to promote tacit $\mathrm{KS}$ and buffer the negative influence of authoritarianism. The extant literature suggests that managers can cultivate their renqing orientation by following the reciprocate rule, placing oneself in others' shoes, and developing empathy [9].

Third, our study confirms that PF perception and IF perception are two important predictors of tacit KS intention. Thus, a work climate that increases fairness perception should be promoted to counteract the negative effects of authoritarianism on tacit $\mathrm{KS}$ intention. The extant literature suggests that PF perception and IF perception can be increased via benevolence, morality, transparency, neutrality, voice, organizational support, two-way communication and leader-member exchange $[7,11,12]$. These management practices can be introduced to increase fairness perception and tacit KS.

\subsection{Limitations}

First, this study is cross-sectional. Although CFA of competing models show that common method bias is an unlike a threat to our results, future research should design longitudinal studies to replicate our research findings. Second, only a Chinese sample is used because this study adopts an emic perspective. However, our research model should be extended to and validated in other countries by conducting a cross-cultural study. Third, we focus on PF perception and IF perception because of their significance. Nevertheless, future research can examine whether the three dimensions of fairness perception will mediate the effects of the three dimensions of paternalistic leadership on tacit KS as well as whether LRO will moderate these mediating processes.

\section{References}

[1] R. J. Bies, J. F. Moag, "Interactional Justice: Communication Criteria of Fairness. In R. J. Lewicki, B. H. Sheppard, \& M. H. Bazerman (Eds.), Research on Negotiations in Organizations (Vol.1, pp. 43-55). Greenwich, CT: JAI Press. 1986.

[2] G. W. Bock, R. W. Zmud, Y.G .Kim, and J.N. Lee, "Behavioral Intention Formation in Knowledge Sharing: Examining the Roles of Extrinsic Motivators, Socialpsychological Forces, and Organizational Climate", MIS Quarterly, 2005, pp.87-111.

[3] S.C.H. Chan, "Paternalistic leadership and employee voice: Does information sharing matter?", Human Relations,2013, pp.1-27.

[4] S. C. H. Chan, X. Huang, E. Snape, and C. K. Lam, "The Janus face of Paternalistic Leaders: Authoritarianism, Benevolence, Subordinates' Organization-based Selfesteem, and Performance", Journal of Organizational Behavior, 2013, 108-128.

[5] X. P. Chen, M. B. Eberly, T. J. Chiang, J. L. Farh, and B. S. Cheng, "Affective Trust in Chinese Leaders: Linking Paternalistic Leadership to Employee Performance", Journal of Management, 2013, pp.796-819.

[6]Y. Chen, R. Friedman, E. Yu, and F. Sun, "Examining the Positive and Negative Effects of guanxi Practices: A Multi-level Analysis of guanxi Practices and Procedural Justice Perceptions", Asia Pacific Journal of Management, 2011, pp. 715-735.

[7] Z. J. Chen, D. Vogel, and Z. H. Wang, "How to Satisfy Citizens? Using Mobile Government to Reengineer Fair Government Processes," Decision Support Systems, 2016, pp.47-57.

[8] B. S. Cheng, L. F. Chou, T. Y. Wu, M. P Huang, and J. L. Farh, "Paternalistic Leadership and Subordinate Responses: Establishing a Leadership Model in Chinese Organizations", Asian Journal of Social Psychology, 2004, pp.89-117.

[9] F. M. Cheung, et al., "Indigenous Chinese Personality Constructs - Is the Five-Factor Model Complete?", Journal of Cross-Cultural Psychology, 2001, pp.407-433.

[10] L. F. Chou, B. S. Cheng, and C. K. Jen, "The Contingent Model of Paternalistic Leadership: Subordinate 
Dependence and Leader Competence", Paper presented at the meeting of the Academy of Management, Honolulu, Hawaii. 2005

[11]J. A. Colquitt, et al., "Justice at the Millennium, a Decade Later: A Meta-Analytic Test of Social Exchange and AffectBased Perspectives", Journal of Applied Psychology, 2013, pp.199-236.

[12] B. Erdogan, "Antecedents and Consequences of Justice Perceptions in Performance Appraisals", Human Resource Management Review, 12, 2002, pp.555-578.

[13] Y.S. Hau, B. Kim, H. Lee, and Y. Kim, "The effects of individual motivations and social capital on employees' tacit and explicit knowledge sharing intentions", International Journal of Information Management, 2013, pp. 356-366.

[14] A. Hayes, Introduction to Mediation, Moderation, and Conditional Process Analysis: A Regression-based Approach. New York: Guilford Publications, 2013.

[15] Q. Huang, R. Davison, and J. Gu, "The Impact of Trust, Guanxi Orientation and Face on the Intention of Chinese Employees and Managers to Engage in Peer-to-Peer Tacit and Explicit Knowledge Ssharing”, Information Systems Journal, 2011, pp.557-577.

[16] Q. Huang, R. M. Davison, H. Liu, and J. Gu, "The Impact of Leadership Style on Knowledge-Sharing Intentions in China", Journal of Global Information Management, 2008, pp. 67-91.

[17] K. K. Hwang, "Face and Favor: The Chinese Power Game", American Journal of Sociology, 1987, pp.944-974.

[18] K.G. Jöreskog , D. Sörbom, LISREL 8: Structural Equation Modeling, Scientific Software International, Chicago, 1996.

[19] G. S. Leventhal, What Should be Done with Equity Theory? New Approaches to the Study of Fairness in Social Relationships. In K. S. Gergen, M. S. Greenberg \& R. H. Willis (Eds.). Social exchange: Advances in theory and research: 27-55. New York: Plenum. 1980.

[20] C. Lin, "To Share or Not to Share: Modeling Tacit Knowledge Sharing, Its Mediators and Antecedents", Journal of Business Ethics, 2007, pp. 411-428.

[21] S. S. Masterson, K. Lewis, B. M. Goldman, and M. S.Taylor, "Integrating Justice and Social Exchange: The Differing Effects of Fair Procedures and Treatment on Work Relationships", Academy of Management Journal, 2000, pp.738-748.

[22] I. Nonaka, "The dynamics theory of organizational knowledge creation", Organization Science, 1994, pp.14-37.

[23] X.J. Ou, R. M. Davison, and L.H.M. Wong, "Using Interactive Systems for Knowledge Sharing: The Impact of Individual Contextual Preferences in China", Information\& Management, 2016, pp.145-156.

[24] P.M. Podsakoff, S.B. Mackenzie, and J.Y. Lee, "Common Method Biases in Behavioral Research: A Critical Review of the Literature and Recommended Remedies", Journal of Applied Psychology, 2003, pp.879-903.

[25] P. Schepers, P. T. van den Berg, "Social Factors of Work Environment Creativity", Journal of Business and Psychology, 2007, pp. 407-428.

[26] P. Sheldon, Y. Q. Li, "Localized poaching and skills shortages of manufacturing employees among MNEs in China”, Journal of World Business, 2013, pp. 186-195.
[27] E. C. Tandoc Jr., P. F. M. Duffy, "Facebook Use, Envy, and Depression Among College Students: Is Face Booking Depressing?", Computers in Human Behavior, 2015, pp.139-146.

[28] S. Wang, R. A. Noe, "Knowledge Sharing: A Review and Directions for Future Research", Human Resource Management Review, 2010, pp.115-131.

[29] M. Wu, X. Huang, C.W. Li, and W. Liu, "Perceived Interactional Justice and Trust-in-supervisor as Mediators for Paternalistic Leadership", Management and Organization Review, 2011), pp. 97-121.

[30] Z. Yan, T. W, Y. Chen, and H. Zhang, "Knowledge Sharing in Online Health Communities: A Social Exchange Theory Perspective", Information\& Management, http: //dx.doi.org /10.1016/ j.im.2016.02.001.

[31] Y. Zhang, M. Y. Huai, and Y. H. Xie, "Paternalistic Leadership and Employee Voice in China: A Dual Process Model", The Leadership Quarterly, 2015, pp.25-36.

[32] X. Zhang, P. de Pablos, and Z. Zhou, "Effect of Knowledge Sharing Visibility on Incentive-based Relationship in Electronic Knowledge Management Systems: An Empirical Investigation", Computers in Human Behavior, 2013 pp. 307-313.

[33] J. Zhou, "A Model of Paternalistic Organizational Control and Group Creativity", Research on Managing Groups and Teams, 2006, pp.75-94.

\section{Appendix}

Authoritarian leadership: 1) My supervisor asks me to obey his/her instructions completely; 2) My supervisor always behaves in a commanding fashion in front of employees. 3) My supervisor determined all decisions in the organization whether they are important or not. 4) In my supervisor's mind, the standard subordinate is an employee who obeys his commands completely. 5) We have to follow his/her rules to get things done. If not, he/she punishes us severely. 6) My supervisor emphasizes that our group must have the best performance of all the units in the organization.

PF perception: 1) The performance evaluation procedure at my organization is a fair one. 2) I am satisfied with the way performance evaluations are done at my organization.

IF perception: 1) My supervisor treated me in a polite manner. 2) My supervisor has treated me with dignity. 3)My supervisor has treated me with respect. 4) My supervisor has refrained from improper remarks or comments.

Tacit KS intention: 1) I intend to share my experience or know-how from work with other organizational members more frequently in the future. 2) I will always provide my know-where or know-whom at the request of other organizational members. 3) I will try to share my expertise from my education or training with other organizational members in a more effective way.

LRO: 1) My supervisor thinks that when dealing with institutions, things can work out more smoothly through the connections of friends working inside. 2) supervisor finds it very hard to say "no" when others make requests.

OCB: 1) I defend my organization when other employees criticize it. 2) I defend my organization when outsiders criticize it. 